\title{
Filtering, Segmentation and Feature Extraction in Ultrasound Evaluation of Breast Lesions
}

\author{
Miguel Alemán-Flores ${ }^{1}$, Patricia Alemán-Flores ${ }^{2}$, Luis Álvarez-León ${ }^{1}$, \\ Rafael Fuentes-Pavón ${ }^{2}$, José M. Santana-Montesdeoca ${ }^{2}$ \\ ${ }^{1}$ Departamento de Informática y Sistemas, \\ Universidad de Las Palmas de Gran Canaria, 35017, Las Palmas, Spain \\ ${ }^{2}$ Sección de Ecografía, Servicio de Radiodiagnóstico, \\ Hospital Universitario Insular de Gran Canaria, 35016, Las Palmas, Spain \\ maleman@dis.ulpgc.es
}

\begin{abstract}
The early diagnosis of breast cancer depends in many cases on the analysis of medical imaging, mainly mammography, ultrasonography and MRI. This work deals with ultrasound images in order to reduce speckle noise, identify the contour of the nodules and analyze a wide range of criteria which allow distinguishing between benign and malignant tumors. We try to automatize the different phases of the process and extract some objective parameters for a robust and reproducible diagnosis. We provide the physicians with both graphical as well as numerical results for the features which have been analyzed.
\end{abstract}

\section{Introduction}

Although mammography is the most widely used modality in breast cancer detection, ultrasonography is a very useful complementary imaging technique which not only provides a different assessment of the lesion, but also allows detecting very small lesions and analyze dense breasts, which is quite difficult using mammography. Radiologists have proved the importance of the information supplied by analyzing a series of criteria on ultrasound images. These include some aspects related to the general shape of the tumor, the relative echogenicity of the different regions and the regularity of the contour [1].

Due to the presence of the characteristic speckle noise, it is quite difficult to perform an accurate automatic segmentation. Therefore, a previous filtering stage is necessary. The information of the contrast as well as the texture of the different regions allow a precise delimitation of the contours. Finally, the automatic analysis of the diagnostic criteria by means of different shape and histogram analysis techniques supplies a deep characterization of the nodules.

Some previous works try to segment the tissues [2] or detect the lesions [3]. Some systems for automatic lesion classification using different features have been proposed $[4,5]$. We intend to carry out a whole process to assess the lesions and support the decision making process. 


\section{Materials and Methods}

We have developed a three-stage process to analyze the ultrasound images. The first phase aims at reducing speckle noise, the second one consists in the segmentation of the nodules and the third one is the analysis of the diagnostic criteria. In order to test the accuracy of the results that we obtain with our techniques, we have used a set of 32 nodules in ultrasound images. Each nodule has been delimited and assessed by 3 experienced specialists. The delimitation has been manually performed twice by each specialist and the evaluation consists in the assessment of a set of diagnostic criteria for each nodule. Furthermore, the biopsy has been performed to determine the malignancy or benignancy of the nodules.

\subsection{Filtering and segmentation}

Due to the characteristic speckle noise of ultrasound images, a noise reducing filter must be first applied before the segmentation. We have tested different filters, including statistical, morphological, iterative or anisotropic filters. The best results have been obtained using a variation of Perona-Malik filter [6] in which we have included texture information obtained from Gabor filters response. From an inner point, a gradient-guided front-propagation algorithm is used to extract an initial pre-segmentation. This can be used as initial contour for the active contour technique [7], for which we apply a level set approach. We have added Gabor-based texture information also in this phase in order to improve the results of the segmentation. Similar approaches are found in [8, 9]. This allows dealing with heterogeneous edges, diffused contours or gradual transitions. Once the segmentation is obtained, we proceed to analyze the features of the nodule.

\section{$2.2 \quad$ Feature Extraction}

The main features which allow discriminating benign from malignant tumors are related to the shape of the nodules, the echogenicity of the different regions and the regularity of their contours. An ellipsoid shape or the presence of two or three gentle and well circumscribed lobulations are considered as benign findings. On the other hand, ramifications or a taller-than-wide shape are considered as malignant findings. Moreover, when examining the smoothness of the contour, if it presents microlobulations, angular margins or spiculation, they are interpreted as malignant findings, as opposite to a rounded and well defined contour. Finally, hyperechogenicity and the presence of a thin echogenic capsule increase the probability of benignancy, whereas calcifications and hypoechogenicity are associated to malignant nodules [1]. In this work, we analyze the global and the local features of the contours as well as the echogenicity histograms, so that some robust, reproducible and accurate measurements can be provided to the specialists in order to perform a more reliable distinction between benign and malignant nodules.

In order to measure how gentle and well circumscribed the contour is, we try to fit to one, two or three overlapping ellipses by means of a gradient descent 
method. The accuracy of the fitting indicates how smooth the contour is and how many lobulations it consists of. Afterwards, we analyze the shape to locate the eventual ramifications of the nodule. Their relative size with respect to the total size indicate their relevance. The local variations of the contour require a detailed analysis of its regularity. We have developed some techniques which involve the search for pseudo-corners, elliptical arcs or high gradient orientation variance and allow locating angular margins, microlobulations or spiculation.

\section{Results}

Figure 1 illustrates some of the graphical results which are provided to the specialists. Table 1 shows the values for the sensitivity, specificity, positive predictive value and negative predictive value for the different criteria. Table 2 presents the corresponding values for the area under the ROC (receiver operating characteristic) curve, which combines sensitivity, specificity and discrimination threshold to provide a more general value.
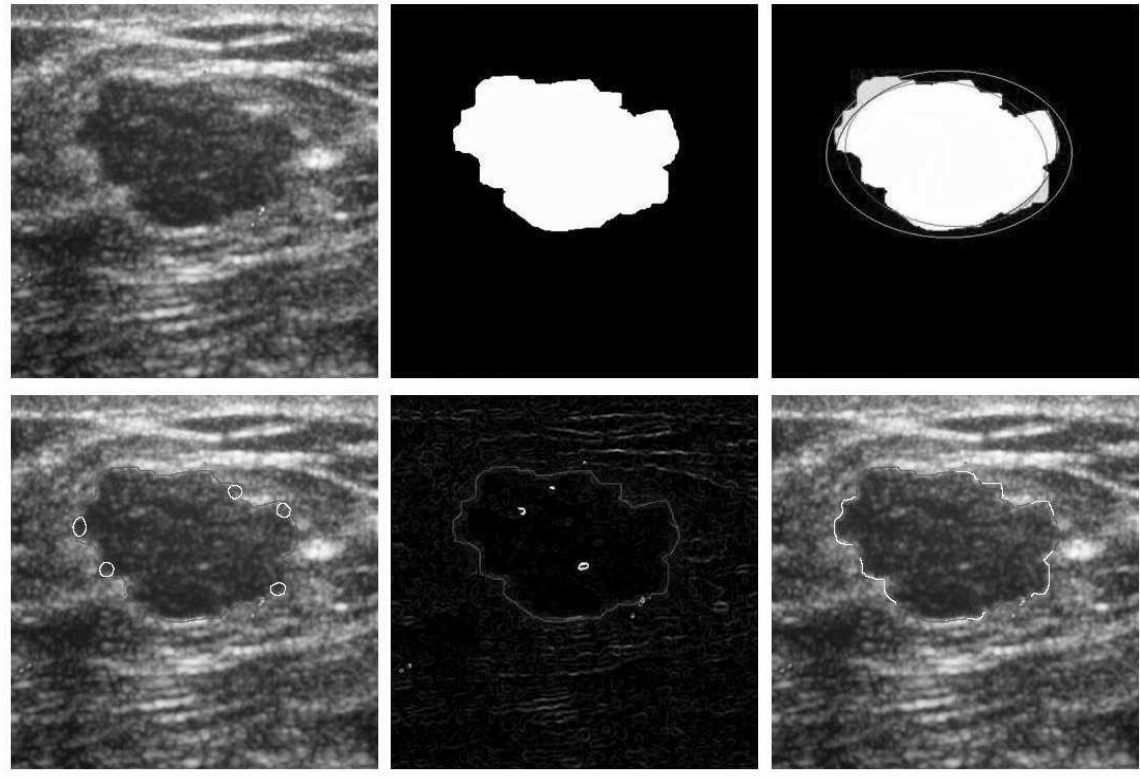

Fig. 1. From top to bottom and left to right: example of a breast nodule, its segmentation and some graphical results for the examination of general shape, microlobulations, calcifications and angular margins 
Table 1. Results: PV $=$ predictive value; $\operatorname{man}=$ assessment of physicians; auto $=$ automatic assessment

\begin{tabular}{lllllllll}
\hline criteria & \multicolumn{2}{c}{ sensitivity } & \multicolumn{2}{c}{ specificity } & \multicolumn{2}{c}{ positive PV } & \multicolumn{2}{c}{ negative PV } \\
& man & auto & man & auto & man & auto & man & auto \\
\hline ellipsoid shape & 92 & 100 & 45 & 37 & 38 & 37 & 94 & 100 \\
gentle lobulations & 100 & 92 & 48 & 37 & 41 & 35 & 100 & 92 \\
capsule & 92 & 30 & 54 & 80 & 42 & 42 & 95 & 75 \\
hyperechogenicity & 100 & 100 & 2 & 2 & 27 & 27 & 100 & 100 \\
hypoechogenicity & 92 & 92 & 20 & 20 & 30 & 30 & 87 & 87 \\
ramifications & 76 & 92 & 82 & 37 & 62 & 35 & 90 & 92 \\
microlobulations & 92 & 100 & 37 & 45 & 35 & 40 & 92 & 100 \\
angular margins & 100 & 92 & 48 & 60 & 41 & 46 & 100 & 95 \\
spiculation & 46 & 53 & 94 & 74 & 75 & 43 & 82 & 81 \\
calcifications & 46 & 30 & 80 & 62 & 46 & 23 & 80 & 70 \\
acoustic shadowing & 23 & 15 & 71 & 80 & 23 & 14 & 71 & 70 \\
\hline
\end{tabular}

Table 2. Results for the area under the ROC curve for the different criteria

\begin{tabular}{ll}
\hline criteria & area under the ROC curve \\
\hline ellipsoid shape & 0.79 \\
gentle lobulations & 0.79 \\
capsule & 0.50 \\
hyperechogenicity & 0.98 \\
hypoechogenicity & 0.99 \\
ramifications & 0.67 \\
microlobulations & 0.51 \\
angular margins & 0.82 \\
spiculation & 0.82 \\
calcifications & 0.50 \\
acoustic shadowing & 0.75 \\
\hline
\end{tabular}

\section{Discussion}

We have presented an automatic process to filter, segment and analyze the features of breast nodules in ultrasound images. The results regarding the sensitivity, specificity, positive predicted value and negative predicted value for single features are comparable to those obtained through the examination of the specialists. The area under the ROC curve shows the importance and robustness of every single feature in the final diagnosis and their relation to the result of the biopsy. We can observe that some automatically extracted parameters are individually precise as well as robust. Since the diagnosis is performed by combining the different criteria and interpreting their relevance, degree and coincidence, we are currently working on a further stage, i.e. a pseudo-expert system which can provide much further information to the radiologists. The results are quite satisfactory and encourage the future work in this research field. 


\section{References}

1. Stavros AT, Thickman D, Rapp CL, et al. Solid breast nodules: Use of sonography to distinguish between benign and malignant lesions. Radiology. 1995;196(1):123-34.

2. Kaufhold J, Chan R, Karl WC, et al. Ultrasound tissue analysis and characterization. Proc SPIE. 1999; p. 73-83.

3. Drukker K, Giger ML, Horsch K, et al. Computerized lesion detection on breast ultrasound. Med Phys. 2002;29(7):1438-46.

4. Rodrigues P, Giraldi G, Provenzano M, et al. A new methodology based on qentropy for breast lesion classification in 3-D ultrasound images. Proc IEEE Eng Ned Biol Soc. 2006; p. 1048-51.

5. Chang RF, Wu WJ, Woo KM, et al. Automatic ultrasound segmentation and morphology based diagnosis of solid breast tumors. Breast Cancer Res Treat. 2005;89(2):179-85.

6. Perona P, Malik J. Scale space and edge detection using anisotropic diffusion. IEEE Trans Pattern Anal Mach Intell. 1990;12(1):629-39.

7. Caselles V, Kimmel R, Sapiro G. Geodesic active contours. Int J Comp Vis. 1997;22(1):61-79.

8. Sagiv C, Sochen N, Zeevi Y. Integrated active contours for texture segmentation. IEEE Trans Image Process. 2006;15(6):1633-46.

9. Chan TF, Vese LA. Active contours and segmentation models using geometric PDE's for medical imaging. In Malladi, R (ed) Geometric Methods in Bio-Medical Image Processing. 2002; p. 63-75. 